BULL. AUSTRAL. MATH. SOC.

VOL. $28(1983), 135-150$.

\title{
VARIATIONAL FORMULATION OF HIGHER ORDER ELLIPTIC BOUNDARY VALUE PROBLEMS
}

\author{
A.J. PRYDE
}

Let $A$ be an elliptic (partial) differential operator of order $2 m$ on a compact manifold $\bar{M}$ with boundary $\Gamma$. Let $B$ be a normal system of $m$ differential boundary operators on $\Gamma$. Assume all manifolds and coefficients are arbitrarily smooth. We construct sesquilinear forms $\mathcal{J}$ in terms of which there are equivalent variational formulations of the natural boundary value problems determined by $A$ and $B$ with solutions in Sobolev spaces $H^{s}(M), 0<s<2 m$. Such forms are also constructed for problems with mixed boundary conditions. The variational formulation permits localization of a priori estimates and the interchange of existence and uniqueness questions between the boundary value problem and an associated adjoint problem.

\section{Introduction}

Let $A$ be a linear elliptic differential operator of order $2 m$ with smooth (complex) coefficients on a smooth n-dimensional compact Riemannian manifold $\bar{M}$ with boundary $\Gamma$. Set $M=\bar{M} \sim \Gamma$ and denote by $H^{S}(M)$ and $H^{S}(\Gamma)$ the corresponding Sobolev spaces of order $s$.

Associated with $A$ are bounded sesquilinear forms

$$
J: H^{m}(M) \times H^{m}(M) \rightarrow \mathbf{C}
$$

Received 30 May 1983. The author wishes to record his gratitude to Alan McIntosh for many useful discussions concerning this work. 
whose associated operators $A_{J}$ agree with $A$ on $C_{0}^{\infty}(M)$ the space of smooth functions with compact support in $M$. The operator

$$
A_{J}: H^{m}(M) \rightarrow \stackrel{\circ}{H}^{-m}(M)
$$

is defined by

$$
\langle A \mathcal{J}, v\rangle=J[u, v]
$$

where $\left\langle\right.$, > denotes the conjugate linear pairing between $H^{m}(M)$ and its (conjugate) dual $\stackrel{\circ}{H}^{-m}(M)$ extending the $L^{2}$ inner product of smooth functions.

For each $m \leq j \leq 2 m-1$ let $C_{j}$ be a normal linear differential boundary operator of order $2 m-j-1$ with smooth coefficients. Associated with $J$ and the $C_{j}$ are unique normal boundary operators $B_{j}$ of order $j$ with smooth coefficients such that for $u, v$ smooth

$$
J[u, v]=(A u, v)-\sum_{j=m}^{2 m-1}\left\langle B_{j} u, C_{j} v\right\rangle
$$

where $($,$) and \langle$,$\rangle denote the inner products in L^{2}(M)$ and $L^{2}(\Gamma)$ respectively.

From the $B_{j}$ and $C_{j}$ one can construct various natural boundary value problems as follows. Firstly, if $X$ is an index set, let $E_{X}=\left\{E_{j}: j \in X\right\}$ when this has meaning. Then for each partition $P^{\prime} \cup Q^{\prime}$ of $\{m, m+1, \ldots, 2 m-1\}$ consider the problem

(1.2) given $f \in L^{2}(M)$ and $t_{P^{\prime}} \in \prod_{j \in P^{\prime}} H^{m-j-\frac{1}{2}}(\Gamma)$ find $u \in H^{m}(M)$ such that $A u=f$ on $M$ and $B_{P^{\prime}} u=t_{P^{\prime}}, C_{Q^{\prime}}, u=0$ on $\Gamma$.

By (1.1) an equivalent variational formulation is

(1.3) given $f \in L^{2}(M)$ and $t_{P^{\prime}} \in \prod_{j \in P^{\prime}} H^{m-j-\frac{1}{2}}(\Gamma)$ find $u \in V$ such that for all $v \in V, J[u, v]=(f, v)-\left\langle t_{P^{\prime}}, C_{P^{\prime}}, v\right\rangle$ 
where $\langle$,$\rangle denotes the pairing on \prod_{j \in P^{\prime}} H^{m-j-\frac{1}{2}}(\Gamma) \times H^{-m+j+\frac{1}{2}}(\Gamma)$ and $V=\left\{v \in H^{m}(M): C_{Q^{\prime}} v=0\right\}$

The boundary conditions $C_{Q}, u=0$ are often called forced or stable, whereas the conditions $B_{P}, u=t_{P^{\prime}}$ are called natural. Since the operators $C_{j}, j \in Q^{\prime}$, have orders $2 m-j-1 \leq m-1$, inhomogeneous boundary conditions $C_{Q^{\prime}} u=t_{Q^{\prime}}$ can be readily replaced by homogeneous ones.

This variational approach was largely developed by Lions. See for example [4]. In [1] Agmon obtained a condition on principal symbols which characterized coercivity for the problem (1.2) when $A$ is strongly elliptic. Grubb [3] obtained a global condition on the operators, when $A$ is properly elliptic and $B_{P^{\prime}}$ and $C_{Q^{\prime}}$ are normal boundary operators, which characterized those problems (1.2) which are associated with a sesquilinear form $J$ as in (1.1). In his book [2], Aubin presents an account of approximation methods for solutions of problems with variational formulation (1.3).

In this paper we consider the problem of determining which normal boundary value problems for an elliptic operator $A$ have a variational formulation via an associated sesquilinear form $J$. We widen slightly the concept of variational formulations (1.3). Following Mclntosh [6] and [7] we consider forms $J$ defined on spaces $V \times W$ where $W$ is not necessarily the same as $V$. This permits the study of a wider class of boundary conditions than the forced and natural conditions of (1.2). In fact we show that all normal boundary value problems for an elliptic operator $A$ have equivalent variational formulations.

Let $s$ be an integer, $I \leq s \leq 2 m-1$, and $P \cup Q$ a partition of $\{0,1, \ldots, 2 m-1\}$, each of $P$ and $Q$ having $m$ elements. If $X=P$ or $Q$ let $X^{\prime}=X \cap\{s, s+1, \ldots, 2 m-1\}$ and $X^{\prime \prime}=X \sim X^{\prime}$. . Let $B_{P}$ be a normal system of differential boundary operators $B_{j}$ of order $j$ with smooth coefficients. Then we consider the following problem 
(1.4) given $f \in L^{2}(M)$ and $t_{P^{\prime}} \in \prod_{j \in P^{\prime}} H^{s-j-\frac{1}{2}}(\Gamma)$ find $u \in H^{S}(M)$ such that $A u=f$ on $M$ and $B_{P^{\prime}} u=t_{P^{\prime}}, B_{P^{\prime \prime}} u=0$ on $\Gamma$.

Extend $B_{P}$ to a normal system $\left\{B_{j}: 0 \leq j \leq 2 m-1\right\}, B_{j}$ of order $j$ with smooth coefficients. Without additional conditions, we construct a boundary system $\left\{C_{j}: 0 \leq j \leq 2 m-1\right\}$ of normal operators $C_{j}$ of order $2 m-j-1$ with smooth coefficients, and a bounded form

$$
J: H^{s}(M) \times H^{2 m-s}(M) \rightarrow \mathbb{C}
$$

such that, for $\operatorname{smooth} u, v$,

$$
J[u, v]=(A u, v)-\sum_{j=m}^{2 m-1}\left\langle B_{j} u, C_{j} v\right\rangle
$$

Let $V=\left\{u \in H^{S}(M): B_{P^{\prime \prime}} u=0\right\}$ and $W=\left\{v \in H^{2 m-s}(M): C_{Q}, v=0\right\}$. Problem (1.4) has the equivalent variational formulation

(1.6) given $f \in L^{2}(M)$ and $t_{P^{\prime}} \in \prod_{j \in P^{\prime}} H^{s-j-\frac{1}{2}}(\Gamma)$ find $u \in V$ such that for all $v \in W, J[u, v]=(f, v)-\left\langle t_{P^{\prime}}, C_{P^{\prime}}, v\right\rangle$.

An important reason for having variational formulations of boundary value problems, especially in the general case where coercivity fails, is that extimates can be localized. Consider for example the following estimate for problem (1.4):

(1.7) $\|u\|_{s} \leq c\left(\|A u\|_{0}+\| B P^{u\|+\| u \|_{s-1}}\right)$ for all $u \in H^{S}(M)$ such that

$$
A u \in L^{2}(M) \text {. }
$$

The norms are the appropriate Sobolev space norms. If $A$ is properly elliptic, $B_{P}$ normal and covering $A$, then (1.7) is satisfied. To prove this (see Lions and Magenes [5]) a similar estimate is proved for $s \geq 2 m$, and then for $s \leq 0$ by duality, and finally for $0<s<2 m$ by interpolation.

The reason for the indirect approach when $0<s<2 m$ is the difficulty of localizing the estimate. Freezing the coefficients of $A$ 
for example is awkward because the space of functions on which (1.7) is to be proved depends on these coefficients. However, with the variational formulation this difficulty is removed and the estimate can be proved directly. Moreover, less smoothness is required of the coefficients of $A$ - smoothness of order $m+|s-m|$ is sufficient, instead of order $2 m$ as required by the interpolation argument. This will be the content of a forthcoming paper in which we solve the problem [5, 2.11.2] posed by Lions and Magenes of finding a proof of (1.7) that avoids interpolation.

Further, using the variational formulation, the question of existence for the boundary value problem can be transferred to the usually simpler question of uniqueness for an adjoint problem. This technique is wellknown. See for example Schechter [11] or Lions and Magenes [5].

The results of this paper will also be used in our work on higher order elliptic equations with mixed boundary conditions. For, suppose $\left\{B_{j}: 0 \leq j \leq 2 m-1\right\}$ is a system of normal differential boundary operators, $B_{j}$ of order $j$ with smooth coefficients. Then we can construct the adjoint system $\left\{C_{j}: 0 \leq j \leq 2 m-1\right\}$ and the form $J$ satisfying (1.5). Suppose $\Gamma_{1}$ and $\Gamma_{2}$ are disjoint open subsets of $\Gamma$ with common smooth boundary $\omega$ satisfying $\Gamma=\Gamma_{1} \cup \Gamma_{2} \cup \omega$. Take $P$ and $Q$ as before. For $1 \leq j \leq 2$ let $r_{j}$ denote restriction to $\Gamma_{j}$. For $1 \leq s \leq 2 m-1$, consider the mixed boundary value problem (1.8) given $f \in L^{2}(M)$, $t_{P^{\prime}} \in \prod_{j \in P^{\prime}} H^{s-j-\frac{1}{2}}\left(\Gamma_{1}\right)$ and

$$
\begin{aligned}
& t_{Q^{\prime}} \in \prod_{j \in Q^{\prime}} H^{s-j-\frac{1}{2}}\left(\Gamma_{2}\right) \text {, find } u \in H^{2}(M) \text { such that } A u=f \text { on } \\
& M, r_{1} B_{P^{\prime}} u=t_{P^{\prime}} \text { and } r_{1} B_{P^{\prime \prime}} u=0 \text { on } \Gamma_{1}, r_{2}{ }_{Q^{\prime}} u=t_{Q^{\prime}} \text { and } \\
& r_{2^{B} Q^{\prime \prime}} u=0 \text { on } \Gamma_{2} .
\end{aligned}
$$

Let $V=\left\{u \in H^{s}(M): r_{1} B_{P^{\prime \prime}}{ }^{u}=0, r_{2} B_{Q^{\prime \prime}} u=0\right\}$ and

$W=\left\{v \in H^{2 m-s}(M): r_{2} C_{P^{\prime}} v=0, r_{1} C_{Q}, v=0\right\}$. Then (1.8) has the equivalent variational formulation 
(1.9) given $f \in L^{2}(M), t_{P^{\prime}} \in \prod_{j \in P^{\prime}} H^{s-j-\frac{1}{2}}\left(\Gamma_{1}\right)$ and

$$
\begin{aligned}
& t_{Q^{\prime}} \in \prod_{j \in Q^{\prime}} H^{s-j-\frac{1}{2}}\left(\Gamma_{2}\right) \text {, find } u \in V \text { such that for all } v \in W, \\
& J[u, v]=(f, v)-\left\langle t_{P^{\prime}}, c_{P^{\prime}}, v\right\rangle-\left\langle t_{Q^{\prime}}, c_{Q^{\prime}}, v\right\rangle .
\end{aligned}
$$

The last two brackets denote respectively the pairings on

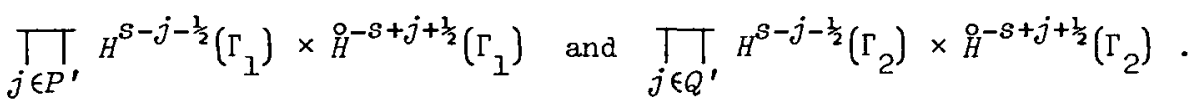

\section{Existence of associated forms}

We make the following assumption on the manifold $M$ :

(2.1) $M$ is an open subset of an $n$-dimensional compact smooth Riemannian manifold $M^{\prime}$ without boundary in which $M$ has $(n-1)$-dimensional smooth boundary $\Gamma$ and closure $\bar{M}$.

Let $\rho(p)$ denote the signed geodesic distance from $\Gamma$ of point $p$ in $M^{\prime}$ near $\Gamma$. We take $\rho>0$ in $M$ and $\rho<0$ in $M^{\prime} \sim \bar{M}$. For some $\varepsilon>0$ there is a neighbourhood $U_{\varepsilon}=\left\{p \in M^{\prime}:-\varepsilon<\rho(p)<\varepsilon\right\}$ of $\Gamma$ with a finite covering by coordinate patches $u_{\varepsilon}^{j}$ with coordinates of the form $x=\left(x^{\prime}, x_{n}\right)$ where $x^{\prime}=\left(x_{1}, \ldots, x_{n-1}\right)$ is a coordinate system in $\Gamma$ and $x_{n}=\rho$.

Define a vector field on $u_{\varepsilon}$ by

$$
n(p)=\left.\frac{\partial}{\partial \rho}\right|_{p} \in T_{p} M^{\prime}
$$

For $p \in \Gamma, n(p)$ is the unit inward normal to $\Gamma$. Define the unit inward conormal at $p \in \Gamma$ to be the unique covector $n(p) \in T_{p}^{*} M^{\prime}$ which annihilates $T_{p} \Gamma$ and is unity on $n(p)$.

The trace operators $\gamma_{j}$ for $j \geq 0$ are defined as usual by $\gamma_{0} u=u \mid \Gamma$ and $\gamma_{j} u=\gamma_{0} D_{n}^{j} u$ where $D_{n}=(1 / i)(\partial / \partial \rho)$.

We assume that 
(2.2) $A=A(p, D)$ is a linear elliptic partial differential operator of order $2 m$ with smooth coefficients on $M^{\prime}$

and

(2.3) $B_{j}=B_{j}(p, D)$ for each $0 \leq j \leq 2 m-1$ is a normal linear partial differential boundary operator of order $j$ with smooth coefficients on $\Gamma$.

If $\sigma\left(B_{j}, p, \xi\right)$ denotes the principal symbol of $B_{j}$ at $(p, \xi) \in T^{*}\left(M^{\prime}\right)$, then $B_{j}$ normal means that $\sigma\left(B_{j}, p, \eta(p)\right)$ is non-zero for each $p \in \Gamma$.

Let $A^{\prime}=A^{\prime}(p, D)$ be the formal adjoint of $A$. That is, $A^{\prime}$ is the unique elliptic operator satisfying

$$
(A u, v)=\left(u, A^{\prime} v\right) \text { for all } u, v \in C_{0}^{\infty}(M) \text {. }
$$

According to Green's theorem (see for example Lions and Magenes [5, Theorem 2.2.1]) there is a uniquely determined adjoint system of differential boundary operators $c_{j}=c_{j}(p, D)$ of orders $2 m-j-1$, $0 \leq j \leq 2 m-1$, such that, for smooth $u, v$,

$$
(A u, v)-\left(u, A^{\prime} v\right)=\sum_{j=0}^{2 m-1}\left\langle B_{j} u, C_{j} v\right\rangle \text {. }
$$

Furthermore, we prove the following result.

THEOREM 2.6. Let $A, B_{j}$ and $M$ satisfy conditions (2.1), (2.2) and (2.3), and let $s$ be an integer, $1 \leq s \leq 2 m-1$. There exists a bounded sesquilinear form $J$ on $H^{s}(M) \times H^{2 m-s}(M)$ such that, for smooth $u, v$,

$$
J[u, v]=(A u, v)-\sum_{j=s}^{2 m-1}\left\langle B_{j} u, C_{j} v\right\rangle .
$$

Before proving the theorem we require the following

LEMMA 2.7. Under the conditions of Theorem 2.6 there exists a bounded sesquilinear form $J$ on $H^{s}(M) \times H^{2 m-s}(M)$ and normal differential boundary operators $F_{j}$ of order $j$ and $G_{j}$ of order $2 m-j-1$ for $0 \leq j \leq 2 m-1$, with smooth coefficients, such that, for smooth $u, v$, 


$$
\begin{aligned}
J[u, v] & =(A u, v)-\sum_{j=s}^{2 m-1}\left\langle F_{j} u, G_{j} v\right\rangle \\
& =\left(u, A^{\prime} v\right)+\sum_{j=0}^{s-1}\left\langle F_{j} u, G_{j} v\right\rangle .
\end{aligned}
$$

Proof. Using a partition of unity, the problem is reduced to constructing $J, F_{j}, G_{j}$ on a coordinate patch $u=u_{\varepsilon}^{i}$ near the boundary and showing that (2.8) holds for $u, v \in \mathcal{C}_{0}^{\infty}(u \cap \bar{M})$.

On $U$ we can write $A$ in the form $A=\sum D^{p} a_{p q} D^{q}$, where $D_{j}=(1 / i)\left(\partial / \partial x_{j}\right)$ and the summation is over multi-indices $p, q$ such that $|p| \leq 2 m-s$ and $|q| \leq s$. Hence $A^{\prime}=D^{q} \bar{a}_{p q} D^{p}$.

Define $J$ on $C_{0}^{\infty}(U \cap \bar{M})$ by

$$
J[u, v]=\int_{\text {unM }} \sum a_{p q}\left(D^{q} u\right) \overline{\left(D^{p} v\right)} .
$$

Integrating by parts we obtain

$$
J[u, v]=\int_{U_{M M}}(A u) \bar{v}+i \int_{\Gamma} \sum_{p, q} \sum_{t=1}^{p_{n}}\left(D_{n}^{t-1} D^{\left(p^{\prime}, 0\right)} a_{p q} D^{q_{u}}\right)^{\overline{D_{n}^{p} n^{-t}} v}
$$

where $p=\left(p_{1}, \ldots, p_{n}\right)=\left(p^{\prime}, p_{n}\right)$. Collecting terms we obtain

$$
J[u, v]=\int_{u n_{M}}(A u) \bar{v}-\int_{\Gamma} \sum_{j=s}^{2 m-1}\left(F_{j} u\right) \overline{\gamma_{2 m-j-1} v}
$$

where $F_{j}$ is a differential operator of order $j$ with smooth coefficients.

The coefficient of $D_{n}^{j}$ in $F_{j}$ is $i a_{\tilde{p} \tilde{q}}$ where $\tilde{p}=(0,2 m-s)$ and $\tilde{q}=(0, s)$. By the ellipticity of $A, a_{\tilde{p} \tilde{q}} \neq 0$ on $M^{\prime}$ and so $F_{j}$ is normal as required for $s \leq j \leq 2 m-1$, as is $G_{j}=\gamma_{2 m-j-1}$. Since $a_{\tilde{p} \tilde{q}}$ is independent of the particular choice of boundary coordinate system, the partition of unity argument preserves normality.

Integrating by parts in the other direction, the second part of (2.8) 
is obtained with $F_{j}=\gamma_{j}$ for $0 \leq j \leq s-1$.

Proof of Theorem 2.6. For $s \leq j \leq 2 m-1, c_{j}=\sum_{t=s}^{2 m-1} c_{j t} \gamma_{2 m-t-1}$ where $c_{j t}$ is a differential operator in the boundary of order $t-j$. We use the convention that the only differential operator of negative order is the zero operator. By (2.5) and (2.8) we have, for smooth $u, v$,

$$
\sum_{j=0}^{2 m-1}\left\langle B_{j} u, c_{j} v\right\rangle=\sum_{j=0}^{2 m-1}\left\langle F_{j} u, G_{j} v\right\rangle
$$

If $y_{j} u=0$ for $0 \leq j \leq s-1$ then

$$
\sum_{j=s}^{2 m-1}\left\langle B_{j} u, C_{j} v\right\rangle=\sum_{t=s}^{2 m-1}\left\langle F_{t} u, G_{t} v\right\rangle
$$

Hence $F_{t}-\sum_{j=s}^{2 m-1} C_{j t}^{\prime} B_{j}$ has normal order at most $s-1$ and can be expressed in the form $\sum_{r=0}^{s-1} S_{t r}^{\gamma}{ }_{r}$ where $S_{t r}$ is a differential operator in the boundary of order $t-r$. It follows that there exists a bounded sesquilinear form $J^{l}: H^{s}(M) \times H^{2 m-s}(M) \rightarrow \mathbf{C}$ such that, for $\operatorname{smooth} u, v$,

$$
\mathcal{J}[u, v]=\sum_{t=s}^{2 m-1} \sum_{r=0}^{s-1}\left\langle S_{t r} \gamma_{p} u, \gamma_{2 m-t-1} v\right\rangle .
$$

This result is proved for the case $s=m$ by Grubb [3, Proposition 5.1]. Her proof readily adapts to our case. If $J^{0}$ denotes a form satisfying (2.8) then $J=J^{0}+J^{I}$ satisfies the claims of the theorem.

\section{Variational formulation}

We now turn to the problem (1.4) posed in the introduction. To use Theorem 2.6, extend $B_{P}$ to a system $\left(B_{j}\right)_{0}^{2 m-1}$ of normal boundary operators $B_{j}$ of order $j$ and let $\left(c_{j}\right)_{0}^{2 m-1}$ be the adjoint system relative to $A$. Let $J^{l}$ be the corresponding sesquilinear form on 
$H^{S}(M) \times H^{2 m-s}(M)$ given by Theorem 2.6. If $J$ denotes the restriction of $J^{l}$ to $V \times W$ then (1.5) holds as required and it follows that (1.4) has the equivalent variational formulation (1.6). We investigate this equivalence more fully.

Let $H_{A}^{s}(M)=\left\{u \in H^{s}(M): A u \in L^{2}(M)\right\}$ with the usual graph norm. Then $B_{j}$ maps $H_{A}^{S}(M)$ to $H^{S-j-\frac{1}{2}}(\Gamma)$ which we abbreviate to $\Pi_{j}$. Thus $B_{P}: H_{A}^{s}(M) \rightarrow \Pi_{P}$ is a bounded operator.

As the orders of $B_{P^{\prime \prime}}$ are less than $s, B_{P^{\prime \prime}}: H^{s}(M) \rightarrow \Pi_{P^{\prime \prime}}$ is also bounded. Since $C_{j}$ has order $2 m-j-1$, if $j \geq s$ then $C_{j}$ maps $H^{2 m-s}(M)$ to $H^{-s+j+\frac{1}{2}}(\Gamma)=\Pi_{j}^{*}$.

If $\stackrel{H}{S}^{S}(M)$ denotes the subspace of $H^{S}\left(M^{\prime}\right)$ consisting of distributions with support in $\bar{M}$ then $\stackrel{\circ}{H}^{S}(M)^{*}=H^{-S}(M)$ with the pairing on $\stackrel{\circ}{H}^{S}(M) \times H^{-s}(M)$ extending by continuity the $L^{2}$ inner product on $C_{0}^{\infty}(M) \times C^{\infty}(\bar{M})$.

Further, by the normality of $c_{j}$, the following is an exact sequence of bounded operators

$$
0 \rightarrow \stackrel{\circ}{H}^{2 m-s}(M) \stackrel{i}{\longrightarrow} W \stackrel{C_{P^{\prime}}}{\longrightarrow} \Pi_{P^{*}}^{*} \rightarrow 0
$$

We use these facts to relate existence/uniqueness or, more generally, Fredholm properties of $\left(A, B_{P}\right)$ with similar properties of the form $J$.

A bounded linear operator between Hilbert spaces is called left semiFredholm, respectively right semi-Fredholm, if it has finite dimensional kernel, respectively finite codimension, and closed range. The operator is Fredholm if both left semi-Fredholm and right semi-Fredholm.

A bounded sesquilinear form $J: V \times W \rightarrow \mathbb{C}$ we will call left semiFredholm, right semi-Fredholm, Fredholm, left invertible, right invertible or invertible if its associated operator $A_{J}: V \rightarrow W^{*}$ defined by $\left\langle A_{J} v, w\right\rangle=J[v, w]$ has the same property. 
Consider the following diagrams, where the subscript ker $A$ denotes the kernel of $A$ in the mentioned space, and $M$ has been dropped from the names of various spaces:
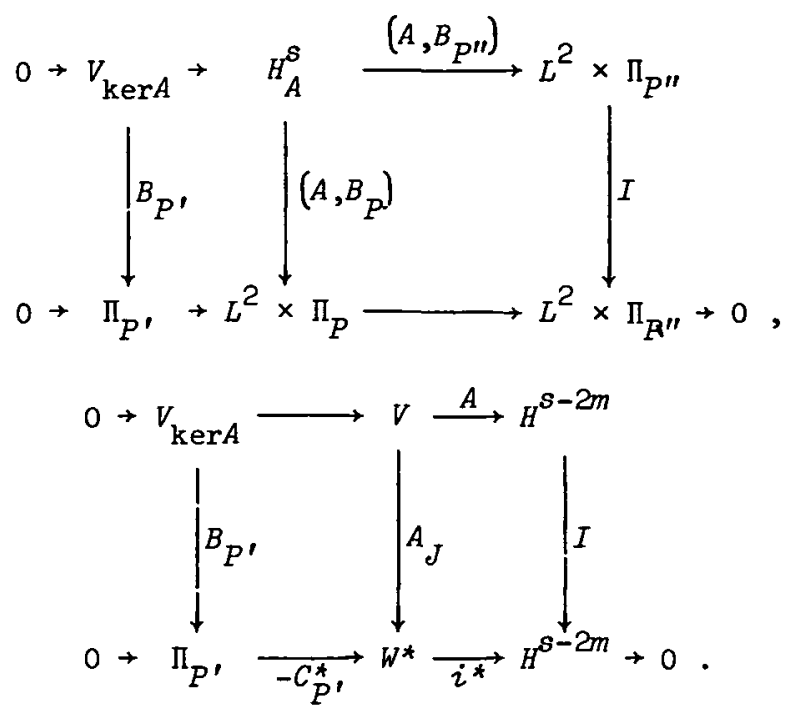

These diagrams commute and the horizontal sequences are exact. The commutativity of (3.3) follows from Theorem 2.6 using the fact that smooth functions are dense in $H^{s}(M), \mathscr{o}^{S}(M)$ and $H_{A}^{s}(M)$. (Lions and Magenes [5].)

If in addition the maps $\left(A, B_{P^{\prime \prime}}\right)$ and $A$ are surjective, which is the case if, for example, the Dirichlet problem for $A$ is uniquely solvable, or more generally if these maps are right semi-Fredholm, then we can apply the five lemma (Pryde [9]) to relate the Fredholm properties of $\left(A, B_{P}\right)$ and $J$.

THEOREM 3.4. Let $A, B_{j}$ and $M$ satisfy conditions (2.1), (2.2) and (2.3), and let $s$ be an integer, $1 \leq s \leq 2 m-1$. If $\left(A, B_{P^{\prime \prime}}\right): H^{s}(M) \rightarrow H^{s-2 m}(M) \times \Pi_{P^{\prime \prime}}$ is right semi-Fredholm, then $\left(A, B_{P}\right): H_{A}^{S}(M)+L^{2}(M) \times \Pi_{P}$ is left semi-Fredholm (right semi-Fredholm) if and only if $J: V \times W \rightarrow \mathbb{C}$ is left semi-Fredholm (right semi-Fredholm). If $\left(A, B_{P^{\prime \prime}}\right)$ is onto then $\left(A, B_{P}\right)$ is left (right) invertible if and only 
If $J$ is left (right) invertible.

Proof. If $\left(A, B_{P^{\prime \prime}}\right): H^{s} \rightarrow H^{s-2 m} \times \Pi_{P^{\prime \prime}}$ is right semi-Fredholm (onto) then so too are $\left(A, B_{P^{\prime \prime}}\right): H_{A}^{s} \rightarrow L^{2} \times \Pi_{P^{\prime \prime}} \quad$ (since $\left.L^{2} \subset H^{s-2 m}\right)$ and $A: V \rightarrow H^{s-2 m}$ (since $\left.V=\operatorname{ker} B_{P^{\prime \prime}}\right)$. The theorem follows from the preceding discussion.

We remark that since $A$ is elliptic, $A: H^{s} \rightarrow H^{s-2 m}$ is right semiFredholm and since $B_{P^{\prime \prime}}$ is normal, $B_{P^{\prime \prime}}: H^{s} \rightarrow \Pi_{P^{\prime \prime}}$ is onto. It follows from the five lemma for example that $\left(A, B_{P^{\prime \prime}}\right): H_{A}^{s} \rightarrow L^{2} \times \mathrm{I}_{P^{\prime \prime}}$ is right semi-Fredholm if and only if $A: V \rightarrow H^{s-2 m}$ is right semi-Fredholm. Hence

COROLLARY 3.5. Let $A, B_{j}$ and $M$ satisfy conditions (2.1), (2.2) and (2.3), and let $s$ be an integer, $1 \leq s \leq 2 m-1$. Then $\left(A, B_{P}\right)$ is Fredholm if and only if $J$ is Fredholm.

This corollary expresses the equivalence, in terms of Sobolev spaces, of the boundary value problem (1.4) and the variational formulation (1.6). Analogous results can be proved to show the equivalence of problems (1.8) and (1.9) for the case of mixed boundary conditions.

\section{Localization}

Because the domain of $J$ depends on $B_{P^{\prime \prime}}$ and $C_{Q^{\prime}}$ it is not suited to localization. We overcome this problem by replacing $J$ with a related form $J^{2}$ whose domain is independent of the operators and which has the same Fredholm properties.

THEOREM 4.1. Suppose $H, K, X, Y$ are Hilbert spaces, $\mathcal{J}: H \times K \rightarrow \mathbf{C}$ is a bounded sesquilinear form and $B: H+X, C: K+Y$ are bounded surjections. Let $J$ be the restriction of $J^{l}$ to $\operatorname{ker} B \times \operatorname{ker} C$ and define $J^{2}$ on $\left(H \times Y^{*}\right) \times\left(K \times X^{*}\right)$ by

$$
\left.J^{2}[(u, \phi),(v, \psi)]=\mathcal{J}[u, v]+i B u, \psi\right\rangle+\langle\phi, C v\rangle .
$$


Then $J$ is left semi-Fredholm, wight semi-Fredholm, left invertible or right invertible if and only if $J^{2}$ has the same property.

Proof. Let $j_{H}: \operatorname{ker} B \rightarrow H$ and $j_{K}:$ ker $C \rightarrow K$ be the injections. Then the following diagrams commute and the horizontal sequences are exact, so the five lemma gives the result immediately:
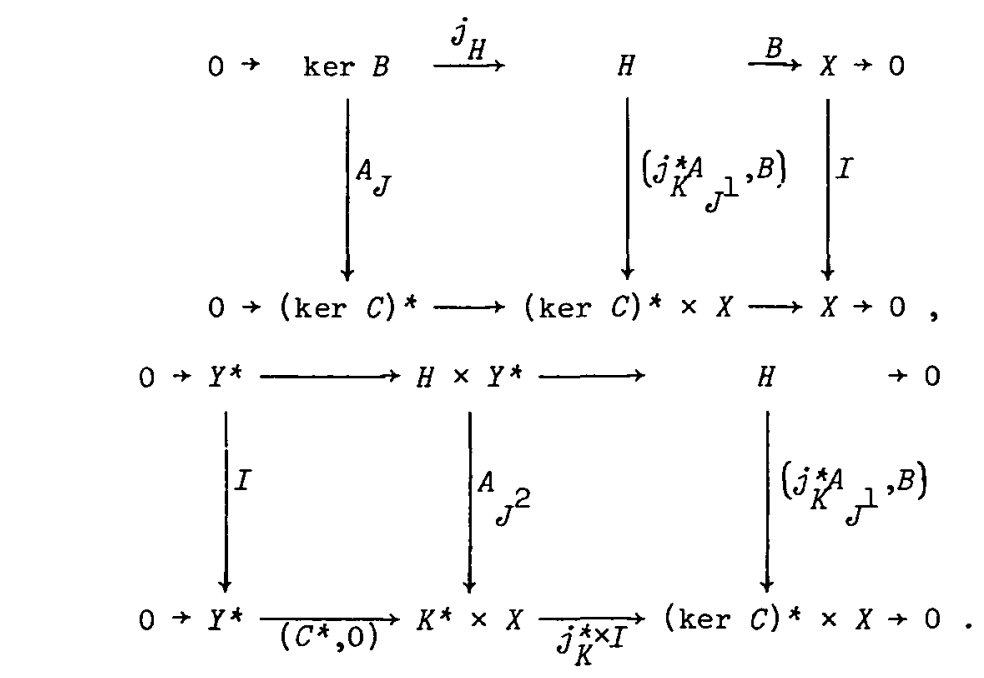

In our case, $\delta^{2}$ on $\left(H^{s}(M) \times \Pi_{Q^{\prime}}\right) \times\left(H^{2 m-s}(M) \times \Pi_{P^{\prime \prime}}^{*}\right)$ is defined by $J^{2}\left[\left(u, \Phi_{Q^{\prime}}\right],\left(v, \psi_{P^{\prime \prime}}\right)\right]=\mathcal{J}^{\mathcal{J}}[u, v]+\left\langle B_{P^{\prime \prime}} u, \psi_{P^{\prime \prime}}\right\rangle+\left\langle\Phi_{Q^{\prime}}, C_{Q^{\prime}}, v\right\rangle$.

Write $u$ for a typical element of $H^{S}(M) \times \Pi_{Q^{\prime}}$ with norm $\|u\|_{S}$ and $\mathbf{v}$ for an element of $H^{2 m-s}(M) \times \Pi_{P^{\prime \prime}}^{*}$ with norm $\|v\|_{2 m-s}$. Let $\|\mathrm{u}\|_{s-1}$ and $\|v\|_{2 m-s-1}$ denote the norms in the larger spaces obtained by replacing each Sobolev space $H^{t}$ by the larger space $H^{t-1}$ in which it is compactly embedded.

By Peetre's lemma [8, p. 728], $d^{2}$ is left semi-Fredholm if and only if

$$
\|u\|_{s} \leq c\left(\sup \frac{\left|\mathcal{J}^{2}[u, v]\right|}{\|v\|_{2 m-s}}+\|u\|_{s-1}\right) \text { for all } u \text {; }
$$


and $J^{2}$ is right semi-Fredholm if and only if

$$
\|v\|_{2 m-s} \leq c \sup \left(\frac{\| \delta^{2}[u, v] \mid}{\|u\|_{s}}+\|v\|_{2 m-s-1}\right) \text { for all } v \text {. }
$$

Using standard localization techniques, these estimates are replaced by similar ones for homogeneous operators with constant coefficients in $\mathbb{R}_{+}^{n}$. In Pryde [10] for example, the localized estimates are shown to be satisfied if and only if $A$ is properly elliptic and covered by $B_{P}$.

\section{Adjoint problems}

Correspondences between the boundary value problem defined by an elliptic $A$ and normel $B_{P}$ and the adjoint problem defined by $A^{\prime}$ and $C_{Q}$ are well known. See for example Schechter [11, Lemma 2.2]. Using the form $J$ and the five lemma, we can readily relate Fredholm properties of the two problems when solutions lie in $H^{S}(M)$ or $H^{2 m-s}(M)$ and $0 \leq \varepsilon \leq 2 m$.

THEOREM 5.1. Let $A, B_{j}$ and $M$ satisfy conditions (2.1), (2.2) and (2.3), and let $s$ be an integer, $1 \leq s \leq 2 m-1$. If $\left(A, B_{P^{\prime \prime}}\right): H^{s}(M) \rightarrow H^{s-2 m}(M) \times \Pi_{P^{\prime \prime}}$ and $\left(A^{\prime}, C_{Q^{\prime}}\right): H^{2 m-s}(M) \rightarrow H^{-s}(M) \times \Pi_{Q^{\prime}}^{*}$ are right semi-Fredholm then $\left(A, B_{P}\right): H_{A}^{s}(M)+L^{2}(M) \times I_{P}$ is left semiFredholm (right semi-Fredholm) if and only if

$$
\left(A^{\prime}, C_{Q}\right): H^{2 m-s}(M)+L^{2}(M) \times \Pi_{Q}^{*}
$$

is might semi-Fredholm (left semi-Fredholm). In particular $\left(A, B_{P}\right)$ is Fredholm if and only if $\left(A^{\prime}, C_{Q}\right)$ is Fredholm.

Proof. Analogous to diagrams (3.1), (3.2) and (3.3) we have the following diagrams:

$$
0 \rightarrow \stackrel{\circ}{H}^{S}(M) \stackrel{i}{\longrightarrow} V \stackrel{B_{Q^{\prime \prime}}}{\longrightarrow} \Pi_{Q^{\prime \prime}}+0,
$$



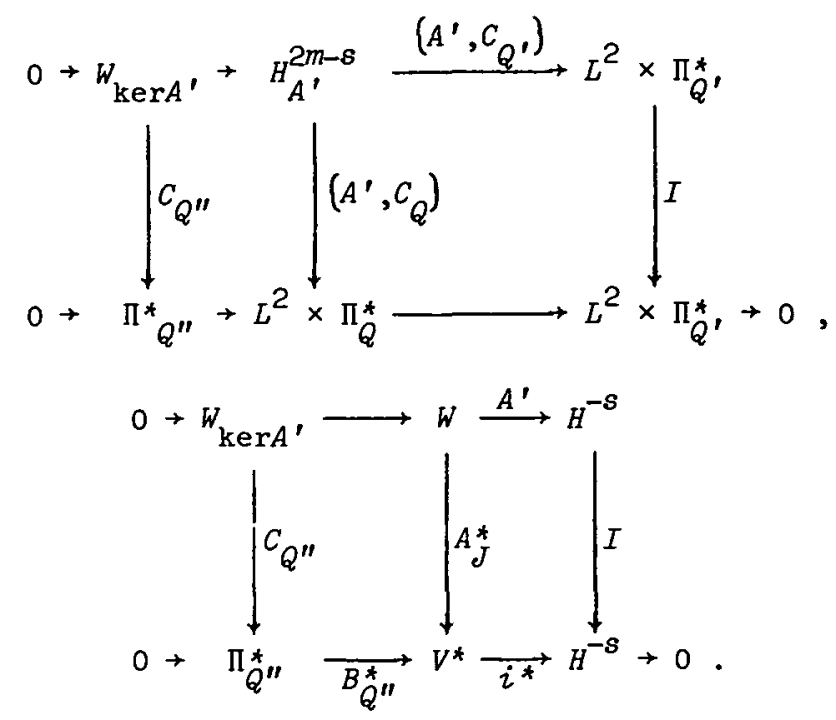

By the same arguments as in Theorem 3.4, $\left(A^{\prime}, C_{Q}\right)$ is right semi-Fredholm (left semi-Fredholm) if and only if $A_{J}^{*}$ is right semi-Fredholm (left semiFredholm) if and only if $\left(A, B_{p}\right)$ is left semi-Fredholm (right semiFredholm). For the last statement of the theorem, compare the proof of Corollary 3.5 .

With regard to the mixed boundary value problem (1.8) one can show similarly that $\left(A, r_{1} B_{P}, r_{2} B_{Q}\right): H_{A}^{s}(M) \rightarrow L^{2}(M) \times r_{1} \Pi_{P} \times r_{2} \Pi_{Q}$ is Fredholm if and only if $\left(A^{\prime}, r_{2} C_{P}, r_{1} C_{Q}\right): H_{A}^{2 m-s}(M) \rightarrow L^{2}(M) \times r_{2} \Pi_{P}^{*} \times r_{1} \Pi_{Q}^{*}$ is Fredholm.

\section{References}

[1] Shmuel Agmon, "The coerciveness problem for integro-differential forms", J. Analyse Math. 6 (1958), 183-223.

[2] Jean-Pierre Aubin, Approximation of elliptic boundary-value problems (Pure and Applied Mathematics, 26. John Wiley \& Sons, New York, London, Sydney, 1972).

[3] Gerd Grubb, "On coerciveness and semiboundedness of general boundary problems", Israel J. Math. 10 (1971), 32-95. 
[4] J.L. Lions, Equations differentielles operationnelles et problèmes aux limites (Die Grundlehren der mathematischen Wissenschaften, 111. Springer-Verlag, Berlin, Gottingen, Heidelberg, 1961).

[5] J.L. Lions et E. Magenes, Problèmes aux limites non homogènes et applications, Volume 1 (Travaux et Recherches Mathematiques, 17. Dunod, Paris, 1968).

[6] Alan G.R. McIntosh, "Bilinear forms in Hilbert space", J. Math. Mech. $19(1969 / 1970), 1027-1045$.

[7] Alan Mclntosh, "Second-order properly elliptic boundary value problems on irregular plane domains", J. Differential Equations 34 (1979), 361-392.

[8] Jaak Peetre, "Another approach to elliptic boundary problems", Comm. Pure Appl. Math. 14 (1961), 711-731.

[9] A.J. Pryde, "The five lemma for Banach spaces", Proc. Amer. Math. Soc. 65 (1977), 37-43.

[10] A.J. Pryde, "Higher order elliptic boundary value problems in spaces with homogeneous norms", J. Austral. Math. Soc. Ser. A 31 (1981), 92-113.

[11] Martin Schechter, "General boundary value problems for elliptic partial differential equations", Comm. Pure Appl. Math. 12 (1959), 457-486.

Department of Mathematics, Institute of Advanced Studies, Australian National University, GPO Box 4, Canberra, ACT 2601, Australia. 